\title{
MUDANÇAS DA ATUAÇÃO MULTIPROFISSIONAL EM PACIENTES COM COVID-19 EM UNIDADES DE TERAPIA INTENSIVA
}

\section{CHANGES IN MULTIPROFESSIONAL PERFORMANCE IN PATIENTS WITH COVID-19 IN INTENSIVE CARE UNITS}

\author{
Hugo Angelo Gomes de Oliveira ${ }^{1}$ \\ Lorena de Medeiros Batista ${ }^{2}$ \\ Alice dos Santos Vasconcelos ${ }^{3}$ \\ Denise Brenda da Silva Fernandes ${ }^{4}$ \\ Ully Dias Nascimento Távora Cavalcanti ${ }^{5}$
}

${ }^{1}$ Cirurgião-Dentista Residente do Programa Multiprofissional em Terapia Intensiva do Real Hospital Português de Beneficência em Pernambuco (COREMU UFPE). Recife, Pernambuco, Brasil. Email: hugoangeloo@gmail.com

${ }^{2}$ Farmacêutica Residente do Programa Multiprofissional em Terapia Intensiva do Real Hospital Português de Beneficência em Pernambuco (COREMU UFPE). Recife, Pernambuco, Brasil. Email: loorenamedeiros@gmail.com

${ }^{3}$ Psicóloga Residente do Programa Multiprofissional em Terapia Intensiva do Real Hospital Português de Beneficência em Pernambuco (COREMU UFPE). Recife, Pernambuco, Brasil. Email: alicevaasconcelos@hotmail.com

${ }^{4}$ Nutricionista Residente do Programa Multiprofissional em Terapia Intensiva do Real Hospital Português de Beneficência em Pernambuco (COREMU UFPE). Recife, Pernambuco, Brasil. Email: denisefnutri@gmail.com

${ }^{5}$ Cirurgião-Dentista, Mestre e Doutora em Odontologia, Preceptora da Residência Multiprofissional em Terapia Intensiva do Real Hospital Português de Beneficência em Pernambuco (COREMU UFPE). Recife, Pernambuco, Brasil. Email: ullydias@gmail.com 


\title{
RESUMO
}

Em dezembro de 2019, o surto do novo coronavírus (SARS-CoV-2) na China se espalhou pelo mundo, tornando-se uma emergência de grande preocupação internacional. A pandemia pelo SARS-CoV-2 fez com que muitos profissionais de saúde modificassem sua rotina de cuidados, especialmente os intensivistas. Este trabalho tem como objetivo avaliar e comparar a atuação multiprofissional em pacientes com COVID-19 internados em unidades de terapia intensiva, antes e após a instalação da pandemia. Foi realizada uma revisão da literatura nas bases de PubCovid-19, PUBMED, EMBASE, MEDLINE, LILACS e Scielo, com artigos publicados de 2015 até o momento, sendo todos os artigos encontrados avaliados, inicialmente, por meio do título e resumo e lidos em sua íntegra apenas aqueles que tratassem do tema. Observou-se a mudança da reconciliação medicamentosa e validação de medicamentos de forma remotas, através de prontuário eletrônico e contato telefônico, bem como o início de atendimentos psicológicos também remotos, aos pacientes, familiares e equipe sendo as intervenções presenciais restritas a casos excepcionais. Além disso, observou-se que pacientes que permanecerem por mais de 48 horas na UTI devem ser considerados em risco de desnutrição.

Palavras-chave: Coronavírus; Odontologia; Farmácia; Nutrição; Psicologia

\begin{abstract}
In December 2019, the outbreak of the new coronavirus (SARS-CoV-2) in China spread across the world becoming an emergency of major international concern. SARS-CoV-2 pandemic resulted in many modifications of health professionals routine care, especially intensivists. This work aims to evaluate and compare the multiprofessional performance in hospitalized patients with COVID-19 in intensive care units, before and after the pandemic scenario. A literature review was carried out on the basis of PubCovid-19, PUBMED, EMBASE, MEDLINE, LILACS and Scielo, with articles published from 2015 to date, with all articles found evaluated initially, by means of the title and abstract and read in its full only those that dealt with the topic. There was a change in medication reconciliation and medication validation remotely, through electronic medical records and telephone contact, as well as the beginning of psychological support, also remote, to patients, family members and staff, with face-to-face interventions being restricted to exceptional cases. In addition, it was observed that patients who remain in the ICU for more than 48 hours should be considered at risk of malnutrition. In addition, elective dental care was suspended, and the use of hydrogen peroxide and povidoneiodine was introduced for mouthwash. Thus, COVID-19 pandemic changed ICU professionals performance due to the new protocols that guide these health professionals in the new context.
\end{abstract}

Keywords: Coronavirus; Dentistry; Pharmacy; Nutrition; Psychology 


\section{INTRODUÇÃO}

No dia 31 de dezembro de 2019, casos de pneumonia de etiologia desconhecida foram identificados na cidade de Wuhan, província de Hubei, na China. Os pacientes com pneumonia apresentaram, principalmente, sintomas clínicos de dispneia, tosse seca, febre e infiltrados pulmonares bilateralmente nas imagens. Todos os casos tinham em comum o Mercado Atacadista de Frutos do Mar de Wuhan, que comercializa peixes e uma variedade de espécies de animais vivos, incluindo morcegos, cobras, marmotas e aves ${ }^{(1)}$. No dia $1^{\circ}$ de janeiro de 2020 , as autoridades de saúde pública de Wuhan fecharam o mercado devido a uma suspeita ligação com o surto ${ }^{(2)}$.

Em 7 de janeiro de 2020, os pesquisadores isolaram rapidamente um novo coronavírus (SARS-CoV-2, também conhecido como 2019-nCoV) de pacientes confirmados infectados com pneumonia. A reação em cadeia da polimerase com transcrição reversa em tempo real (RTPCR) e o sequenciamento de última geração foram utilizados para caracterizá-la. Em 23 de janeiro de 2020, devido ao grande fluxo de pessoas durante o Festival da Primavera na China, o transporte público foi suspenso em Wuhan e, eventualmente, em todas as cidades da província de Hubei para reduzir o risco de novas transmissões ${ }^{(2)}$.

Em 30 de janeiro de 2020, a OMS declarou que o surto chinês de COVID-19 era uma emergência de saúde pública de interesse internacional, representando um alto risco para países com sistemas de saúde vulneráveis. O comitê de emergência declarou que a disseminação da COVID-19 pode ser interrompida pela detecção precoce, isolamento, tratamento imediato e implementação de um sistema robusto para rastrear contatos ${ }^{(3)}$. A síndrome respiratória aguda grave (SARS) é uma doença infecciosa recentemente emergente causada pelo novo coronavírus. A fatalidade geral da SARS é de cerca de $10 \%$ na população geral, sendo mais de $50 \%$ em pacientes com 65 anos ou mais ${ }^{(4)}$. 
A pandemia de SARS-CoV-2 propôs um novo desafio para os profissionais de saúde, especialmente os intensivistas. Cerca de 15 a 20\% dos pacientes suspeitos e confirmados com infecção por SARS-CoV-2 com febre desenvolveram hipoxemia grave (desde a segunda semana do curso da doença) e necessitaram de algum tipo de suporte ventilatório, como cânula nasal de alto fluxo e ventilação mecânica não invasiva e invasiva. Além disso, podem ocorrer outras complicações, incluindo choque, lesão renal aguda, sangramento gastrointestinal e rabdomiólise. O manejo de pacientes gravemente enfermos com infecção por SARS-CoV-2 requer uma carga de trabalho notável para a equipe multiprofissional na terapia intensiva ${ }^{(5,6)}$. Nesse contexto, as Unidades de Terapia Intensiva (UTI) são o local de referência para prestar cuidados críticos, especializados e ininterruptos, contando com uma equipe multiprofissional, destinada a atender pacientes graves e recuperáveis ${ }^{(7)}$. Além dos cuidados voltados ao tratamento direto ao organismo doente, entende-se que a internação em uma UTI gera alterações e desencadeia o aparecimento de estressores na vida do paciente e de seus familiares ${ }^{(8)}$.

Sendo assim, este trabalho tem como objetivo avaliar e comparar a atuação multiprofissional em pacientes com COVID-19 internados em unidades de terapia intensiva, antes e após a instalação da pandemia, em hospitais públicos ou privados a fím de um cuidado integral ao paciente gravemente enfermo.

\section{MÉTODOS}

Trata-se de uma revisão da literatura a partir de fontes secundárias das plataformas: PubCovid-19, a qual está indexada na Biblioteca Nacional de Medicina dos Estados Unidos (PUBMED); Banco de dados Excerpta Medica (EMBASE); MEDLINE; LILACS e; Scielo. A pesquisa foi realizada durante o mês de julho de 2020, sendo todos os artigos encontrados avaliados, inicialmente, por meio do título e resumo e lidos em sua íntegra apenas aqueles que tratassem sobre o tema. Para a realização da busca foram utilizados os seguintes descritores em 
inglês: "Intensive Care Units"; "Covid-19"; "Coronavirus"; "SARS Virus", "Dentistry"; "Pharmaceutical Services"; "Nutrition Therapy" e "Psychology". Os critérios de inclusão definidos para a seleção dos artigos foram: Artigos publicados em português ou inglês; Artigos na íntegra que retratassem a temática; Atividades desenvolvidas na UTI; Protocolo de manejo com pacientes com COVID-19 e; Artigos publicados e indexados nos referidos bancos de dados a partir de 2015 até o momento.

\section{RESULTADOS}

A partir da leitura dos artigos selecionados, dividiu-se em quatro grandes áreas (Farmácia, Nutrição, Odontologia e Psicologia) sendo levantadas as principais atuações e mudanças encontradas nas UTIs relacionadas ao contexto da COVID-19, contribuindo para a qualidade de vida dos pacientes e uma melhora no serviço. Todos esses dados, podem ser observados na tabela 1.

Tabela 1: Principais atuações da farmácia, nutrição, odontologia e psicologia no paciente internado em unidades de terapia intensiva e mudanças após a COVID-19.

\begin{tabular}{ll}
\hline Área & Principais atuações \\
\hline
\end{tabular}




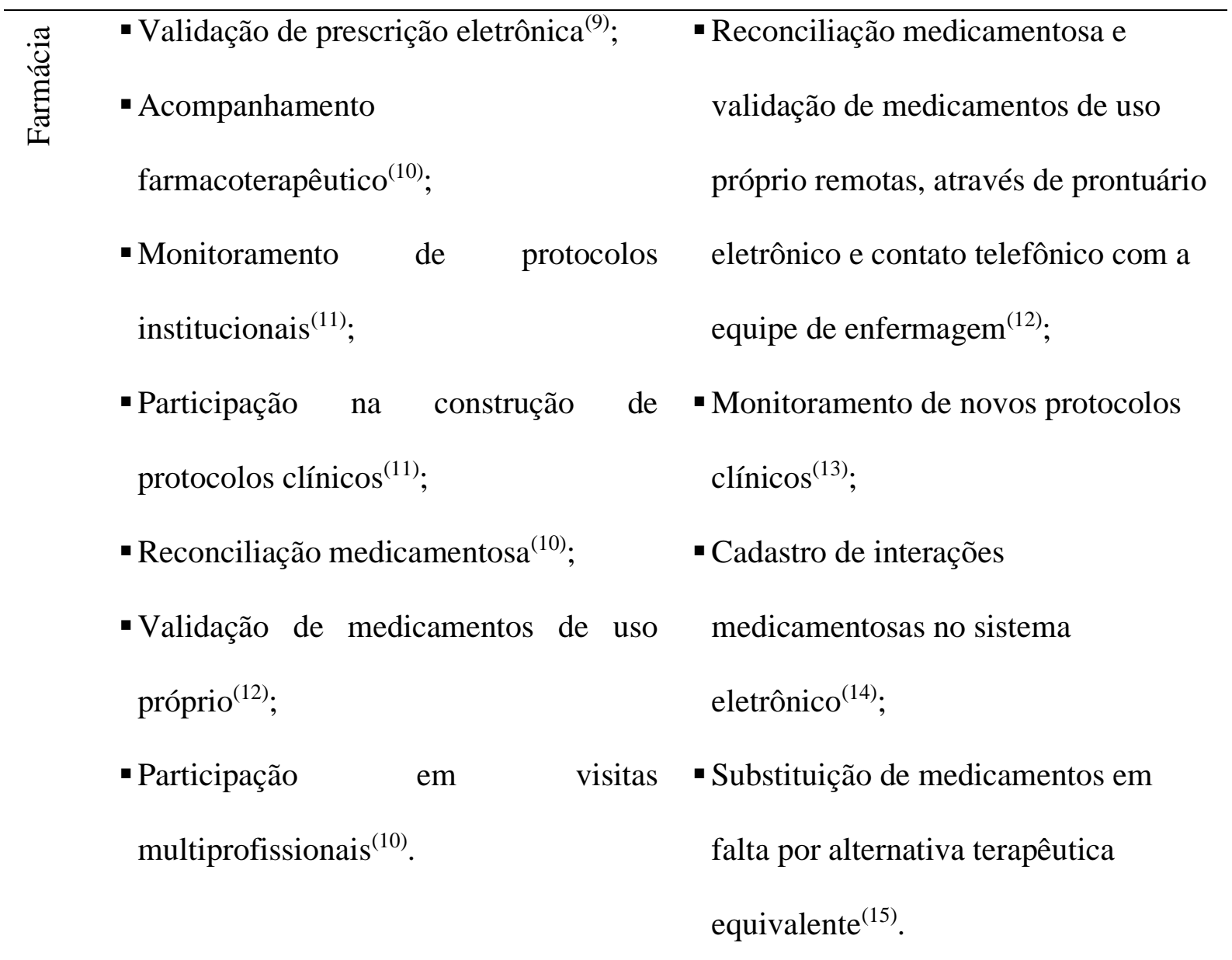




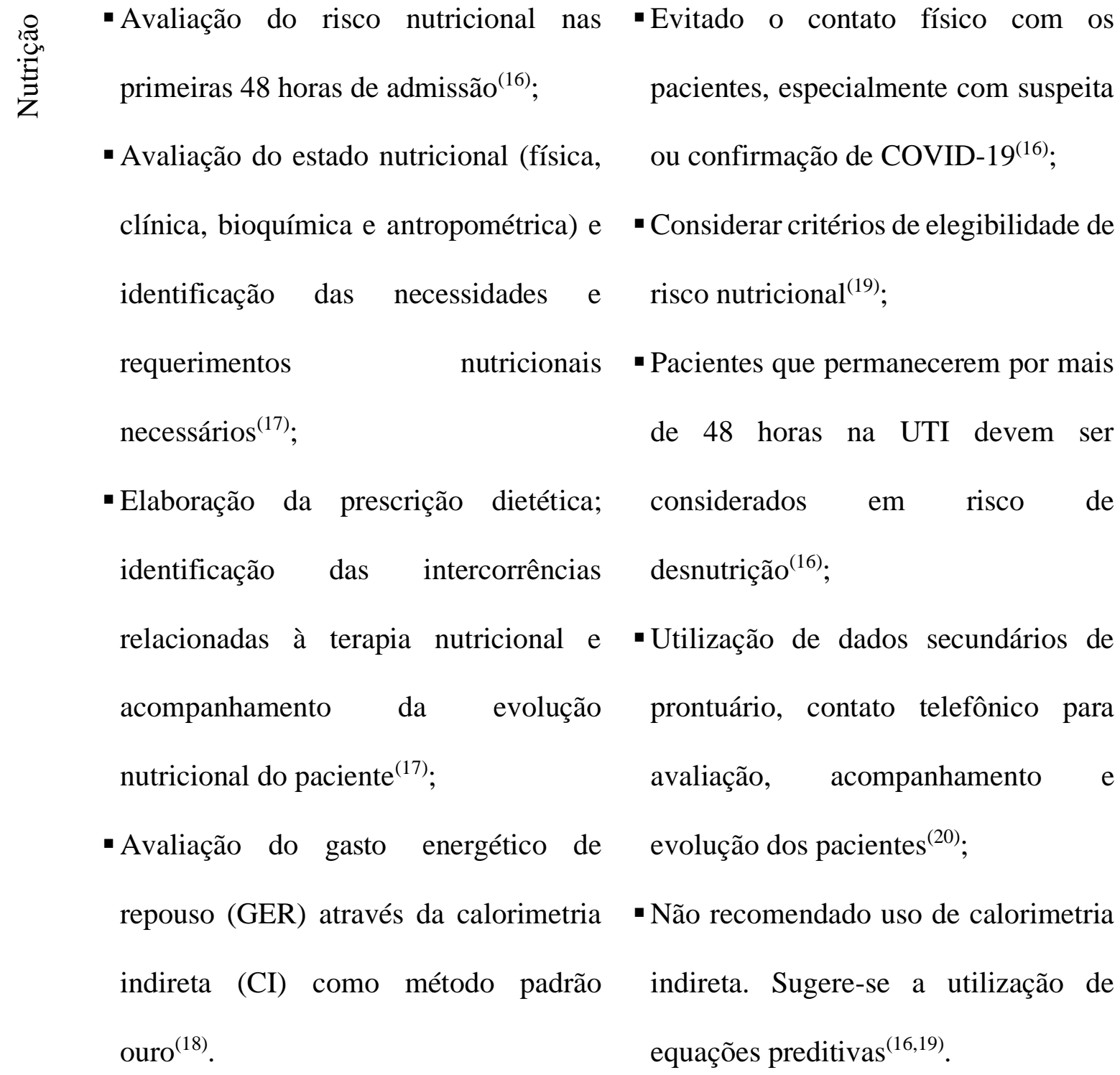


- Melhoria da qualidade de vida dos - Tratamentos odontológicos eletivos pacientes através da eliminação de focos $\operatorname{postergados}^{(27)}$;

de infecção ${ }^{(21)}$;

- Uso do peróxido de hidrogênio e

- Prevenção, diagnóstico e tratamento de iodopovidona para bochechos ${ }^{(28,29)}$; lesões em boca ${ }^{(22)}$;

- Remoção mecânica do biofilme com

- Tratamento de lesões traumáticas em

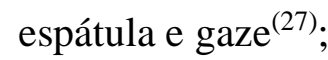
pacientes $^{(23)}$;

- Proibição de dispositivos protéticos

- Implementação de protocolos de higiene $\operatorname{armazenados~no~hospital~}^{(27)}$; bucal $^{(24,25)}$;

- Protocolos rígidos

de

- Prevenção da pneumonia associada a biossegurança $^{(23)}$; ventilação mecânica ${ }^{(26)}$.

- Uso de máscara N95 ou PFF2 em procedimentos geradores de aerossóis $^{(27)}$;

- Uso de capote ou avental impermeável com mangas longas, punho de malha ou elástico e abertura posterior ${ }^{(27)}$. 


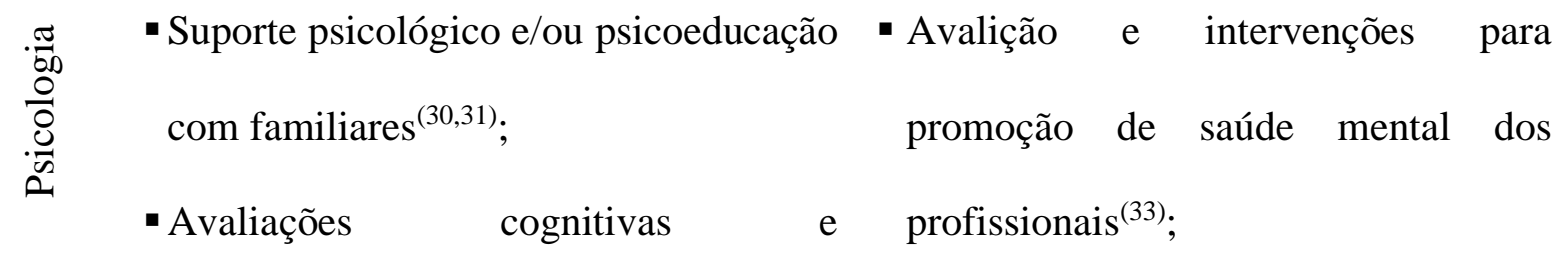
neuropsicológicas ${ }^{(30)}$ - Atendimentos psicológicos remotos,

- Assistência psicológica ao paciente, aos pacientes, familiares e equipe, por com intervenções de apoio e meio de tecnologia da comunicação

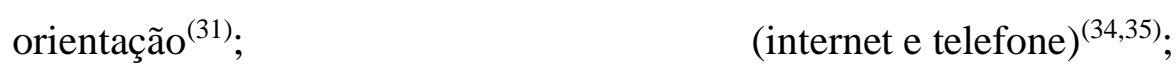

- Mediação da tríade paciente-família- - Intervenções presenciais restritas à equipe $^{(31)}$; $\quad$ casos excepcionais ${ }^{(35)}$;

- Intervenções grupais $^{(31)}$;

- Suporte e orientação aos pacientes,

- Escuta clínica ${ }^{(32)}$. familiares e equipe para manejo de situações de estresse, estratégias de enfrentamento e autocuidado $^{(35)}$;

- Visitas à área de descanso dos profissionais para escuta clínica ${ }^{(35)}$;

- Intervenções junto a pacientes COVID-19 em desmame ventilatório $^{(36)}$.

Fonte: Autoria própria

\section{DISCUSSÃO}

A UTI é definida como uma área de convergência multiprofissional voltada para o atendimento de pacientes com potencial ou efetivo comprometimento das funções vitais decorrentes de falhas de um ou mais sistemas orgânicos ${ }^{(37)}$. Pacientes sob cuidados intensivos 
constituem um grupo de indivíduos em condições graves que requer uma equipe multidisciplinar qualificada destinada à assistência e atenção à saúde ${ }^{(38)}$.

O farmacêutico no âmbito hospitalar atua em toda a cadeia medicamentosa, desde a aquisição do medicamento até a administração segura no paciente, sendo assim, uma função essencial é a participação na Comissão de Farmácia e Terapêutica, atuando na seleção e padronização de medicamentos, na elaboração de diretrizes clínicas e protocolos terapêuticos, na promoção de informações sobre medicamentos e outros produtos para saúde assim como na participação de estudos de custo-efetividade. Além disso, acompanhar a farmacoterapia do paciente através da validação de prescrições e participação em visitas multiprofissionais para intervir, quando necessário, no ajuste de doses de medicamentos, frequência de uso, adequação da via de administração, adequação da forma farmacêutica, duração da terapia, entre outros aspectos, com a finalidade de garantir a segurança e a eficácia da terapia medicamentosa do paciente, resultando em uma recuperação mais rápida com consequente diminuição do tempo de internamento ${ }^{(11)}$. Também é de suma importância o controle de estoques e abastecimento de medicamentos e produtos médico-hospitalares ${ }^{(15)}$.

A farmácia hospitalar vem enfrentando diversos desafios frente ao cenário da pandemia e, para continuar ofertando um serviço de qualidade à população, foi necessária a adoção de algumas estratégias de enfrentamento e adaptação de algumas atividades ${ }^{(12)}$. O rápido crescimento no número de casos graves teve como consequência o aumento repentino na demanda por alguns medicamentos, resultando em uma crise na cadeia de produção e distribuição com consequente dificuldade de aquisição, o que levou a uma necessidade de um novo planejamento para abastecimento e à busca por alternativas terapêuticas ${ }^{(15,39)}$. A falta de um tratamento específico também revelou o papel essencial do farmacêutico no monitoramento de novos protocolos clínicos estabelecidos na comunidade científica com o uso off-label de algumas drogas, para garantia do uso racional de medicamentos e segurança do paciente ${ }^{(13)}$. 
As atividades de reconciliação medicamentosa e validação de medicamentos de uso próprio sofreram adaptações e passaram a acontecer de forma remota, via prontuário eletrônico e contato telefônico com a equipe de enfermagem ${ }^{(12)}$. A adequação de posologias de alguns medicamentos, quando possível, também pode ser realizada a fim de minimizar a exposição dos profissionais ${ }^{(14)}$.

No contexto hospitalar, especificamente em UTI, o nutricionista atua de modo a ofertar uma melhor assistência nutricional ao paciente, através de avaliação do estado nutricional; elaboração de prescrição dietética e identificação das intercorrências relacionadas à terapia nutricional, realizando o acompanhamento da evolução nutricional do paciente. As intervenções nutricionais individualizadas e precoces estão ligadas à diminuição do tempo de internação hospitalar, aumento da oferta calórico-proteica, diminuição da mortalidade e melhoria da qualidade de vida após a alta ${ }^{(17,40)}$.

Se tratando de pacientes com COVID-19, por apresentarem quadro clínico caracterizado principalmente por complicações respiratórias agudas, requerem tratamento em UTI, na qual o tempo de permanência, polimorbidade e idade avançada são fatores comumente associados ao alto risco de desnutrição. A prevalência de risco nutricional é conhecida, e em grande parte associada a ausência de mobilidade devido alterações musculoesqueléticas em decorrência do hipercatabolismo, bem como aumento das necessidades nutricionais e diminuição da ingestão $\operatorname{alimentar}^{(41,42)}$.

O rastreio do risco e avaliação nutricional devem ser realizados precocemente, visando ao desenvolvimento de plano de cuidados e estratégias nutricionais, monitorando sua eficácia. O manejo nutricional é, em princípio, muito semelhante a qualquer outro paciente de UTI admitido com comprometimento pulmonar, visando à manutenção e / ou recuperação da massa muscular e prevenção de complicações relacionadas ao internamento e desnutrição $\operatorname{prolongada}^{(43,44)}$. 
A Odontologia se destaca pelo seu papel interprofissional e multidisciplinar, contribuindo para a integralidade do cuidado e busca da saúde global e da melhoria da qualidade de vida de pacientes hospitalizados ${ }^{(45)}$. Atualmente, as infecções hospitalares são uma das principais causas de mortalidade e morbidade dos pacientes internados ${ }^{(46)}$. Considerações importantes têm sido relatadas em estudos onde apontam o biofilme bucal como destaque para o desenvolvimento de infecções bacterianas, virais e/ou fúngicas, especialmente em pacientes internados em $\mathrm{UTI}^{(47)}$.

Segundo a Associação de Medicina Intensiva Brasileira (AMIB), a manutenção da higiene bucal para prevenção de pneumonia associada à ventilação mecânica (PAVM) adotada pelas instituições hospitalares com a solução de clorexidina a $0,12 \%$ deve ser mantida com o intuito de evitar novos casos de pneumonia por infecção de microrganismos que não o SARSCoV-2. O uso de substâncias oxidantes, com o intuito de inativar o vírus presente na saliva, como o peróxido de hidrogênio a $1 \%$, pode ser utilizada para o SARSCoV-2, a depender de discussões multidisciplinares com outros membros da equipe assistencial ${ }^{(27)}$.

Além dos procedimentos odontológicos de urgência, como sangramentos orais associados aos medicamentos e ressecamento bucal/labial em função dos respiradores, devem ser acrescentadas outras recomendações pertinentes aos pacientes oncológicos e sistemicamente comprometidos, como: Tratamento de mucosites orais com indicação de laserterapia; Tratamento odontológico necessário prévio a procedimento médico crítico; Biópsia de desordens potencialmente malignas; Instalação de protetores bucais em pacientes entubados em UTI com trauma nos tecidos moles e tubo orotraqueal; Tratamento de lesões traumáticas em pacientes entubados em UTI e; Reembasamento de prótese total para progressão de dieta oral. Seguindo todos os protocolos de biossegurança para o atendimento a este grupo de pacientes minimizando o risco de contaminação cruzada ${ }^{(23)}$. 
A Psicologia no contexto hospitalar tem o referencial teórico clínico como um de seus pilares e possui a tarefa de endereçar-se e oferecer espaço de escuta às condições subjetivas que atravessam a experiência de hospitalização do sujeito e das pessoas ao seu redor ${ }^{(48)}$. Na UTI, o papel do psicólogo consiste em participar de forma ativa do cuidado multiprofissional ao atuar na assistência psicológica ao paciente, atentando e escutando seus aspectos emocionais diante da internação e quadro clínico; junto aos familiares, ofertando acolhimento, suporte e escuta; junto aos profissionais, possibilitando espaços para expressão emocional; e mediando as relações da tríade paciente-família-equipe ${ }^{(31)}$.

A psicologia na terapia intensiva foi, então, convocada a atuar junto aos profissionais que compõem a linha de frente do combate à COVID-19 e junto aos pacientes e familiares que vivenciam as angústias potencializadas pelo adoecimento, a hospitalização e o isolamento social. Nesse sentido, foi preciso construir as diretrizes de um setting terapêutico que respondesse à urgência dos sujeitos em seus atravessamentos atuais. A resposta psicológica ao sofrimento psíquico presente no contexto da terapia intensiva e potencializado pela pandemia não poderia ser interrompida, mas adaptada e ampliada, levando em conta a biossegurança e as demandas de saúde mental. Destarte, a atuação do psicólogo na UTI teve como principal mudança a priorização de plataformas online e/ou telefônicas para oferta de serviços psicológicos $^{(49)}$.

Tendo em vista esse contexto, o Conselho Federal de Psicologia publicou a Resolução $\mathrm{n}^{\mathrm{o}} 4 / 2020$ suspendendo as disposições contrárias anteriores e permitindo que o profissional cadastrado no "e-Psi" faça a prestação de serviços através de tecnologia da informação e comunicação. Embora necessárias para o momento, essas modalidades de atendimento configuram um desafio à psicologia hospitalar, tanto em seus aspectos éticos, quanto teóricos e técnicos e requerem que haja reflexão e aprofundamento teórico acerca de seu uso ${ }^{(50)}$. 


\section{CONCLUSÃO}

Diante dos fatos, a COVID-19 é uma doença grave contagiosa que vem causando a morte de milhares de pessoas no mundo. A pandemia da COVID-19 mudou a forma das profissões atuantes nas UTIs, citadas no estudo, devido aos novos protocolos que norteiam esses profissionais da área de saúde na situação de pandemia, reforçando os benefícios de atuação interprofissional em unidades de terapia intensiva.

Sendo assim, percebe-se que a literatura sobre esta temática ainda se encontra em sua fase embrionária e exploratória, sendo predominantemente originária de países que vivenciaram a pandemia antes do Brasil. Esta realidade nos convoca a pensar e produzir estudos que considerem o contexto das unidades de terapia intensiva brasileiras e forneçam parâmetros para a atuação multiprofissional na UTI em meio à pandemia.

\section{REFERÊNCIAS}

1. Lu H, Stratton CW, Tang YW. Outbreak of pneumonia of unknown etiology in Wuhan, China: The mystery and the miracle. J Med Virol. 2020;92(4):401-2.

2. Zhu N, Zhang D, Wang W, Li X, Yang B, Song J, et al. A novel coronavirus from patients with pneumonia in China, 2019. N Engl J Med. 2020;382(8):727-33.

3. Organization WH. Novel Coronavirus (2019-nCoV) [Internet]. 2020. Available from: https://covid19.who.int/region/searo/country/id

4. Du L, He Y, Zhou Y, Liu S, Zheng B-J, Jiang S. The spike protein of SARS-CoV - A target for vaccine and therapeutic development. Nat Rev Microbiol. 2009;7(3):226-36.

5. WHO. World Health Organization. 2020.

6. Devereaux A, Christian MD, Dichter JR, Geiling JA, Rubinson L. Summary of 
suggestions from the Task Force for Mass Critical Care Summit, January 26-27, 2007. Chest. 2008;133(5 SUPPL.):1S-7S.

7. Marques IR, Souza AR de. Tecnologia e humanização em ambientes intensivos. Rev Bras Enferm. 2010;63(1):141-4.

8. Bitencourt AGV, Neves FBCS, Dantas MP, Albuquerque LC, Melo RMV de, Almeida A de M, et al. Análise de estressores para o paciente em Unidade de Terapia Intensiva. Rev Bras Ter Intensiva. 2007;19(1):53-9.

9. Jourdan JP, Muzard A, Goyer I, Ollivier Y, Oulkhouir Y, Henri P, et al. Impact of pharmacist interventions on clinical outcome and cost avoidance in a university teaching hospital. Int J Clin Pharm [Internet]. 2018;40(6):1474-81. Available from: https://doi.org/10.1007/s11096-018-0733-6

10. Bosma BE, van den Bemt PMLA, Melief PHGJ, van Bommel J, Tan SS, Hunfeld NGM. Pharmacist interventions during patient rounds in two intensive care units: Clinical and financial impact. Neth J Med. 2018;76(3):115-24.

11. BRASIL. Resolução nº 449. Cons Fed Fermácia. 2006;(November):1-21.

12. Santolaya-Perrin R, García-Martín Á, Carrero-Fernández A, Torres-Santos-Olmo R. Hospital pharmacist experience in a multidisciplinary team in special care settings. Farm Hosp. 2020;44(7):36-9.

13. Bermejo-Vicedo T, Queralt Gorgas M. COVID-19 Pandemic. New challenge for hospital pharmacy services. Farm Hosp. 2020;44(7):3-4.

14. Gil-Navarro MV, Luque-Márquez R. Hospital Pharmacy in the multidisciplinary team of COVID inpatient units. Farm Hosp. 2020;44(7):40-2.

15. Shuman AG, Fox ER, Unguru Y. COVID-19 and Drug Shortages: A Call to Action. J 
Manag care Spec Pharm [Internet]. 2020;26(8):945-7. Available from: http://www.ncbi.nlm.nih.gov/pubmed/32715960

16. Campos LF, Barreto PA, Ceniccola GD, Gonçalves RC, de Matos LBN, Zambelli CMSF, et al. Parecer BRASPEN/AMIB para o enfrentamento da COVID-19 em pacientes hospitalizados. Braspen J. 2020;35(1):3-5.

17. Silva MTG da, Oliveira MM e. The importance of the nutritional therapy at the Intensive Care Unity. Braspen J [Internet]. 2016;31(4):347-56. Available from: http://www.braspen.com.br/home/wp-content/uploads/2017/02/12-AO-A-importânciada-terapia-nutri.pdf

18. Brazilian Society of Parenteral and Enteral Nutrition. Diretriz Brasileira de Terapia Nutricional Domiciliar. Braspen J. 2018;1(2525-7374):37-55.

19. Piovacari SMF, Santos GFCG, Santana GA, Scacchetti T, Castro MG. Flow of nutritional assistance for patients admitted with COVID-19 and SCOVID-19 in a hospital unit. Braspen J. 2020;35(1):6-8.

20. Brasil. Boas Práticas Para a Atuação Do Nutricionista E Do Técnico Em Nutrição E Dietética Durante a Pandemia Do Novo Coronavírus (Covid-19). Cons Fed e Reg Nutr. 2020;3:15.

21. Tunes RS, Tunes U. Hospital Dentistry: state of the art. J Dent Pub H. 2018;9(1):5-7.

22. Fernandes IS, Fraga CPT. Cavity of Oncological Treatment of Head and Neck Revista Científica UMC. Rev Científica UMS. 2019;4(1):1-16.

23. Franco JB, Camargo AR de, Perres MPS de M. Cuidados Odontológicos na era do COVID-19 : recomendações para procedimentos odontológicos e profissionais. Vol. 74, Rev Assoc Paul Cir Dent. 2020. p. 18-21. 
24. da Silva JL, El Kadre GDA de O, Kudo GAH, Junior JFS, Saraiva PP. Oral health of patients hospitalized in the intensive care unit. J Contemp Dent Pract. 2016;17(2):1259.

25. Blum DFC, Munaretto J, Baeder FM, Gomez J, Castro CPP, Bona Á Della. Influence of dentistry professionals and oral health assistance protocols on intensive care unit nursing staff. A survey study. Rev Bras Ter Intensiva. 2017;29(3):391-3.

26. Ory J, Mourgues C, Raybaud E, Chabanne R, Jourdy JC, Belard F, et al. Cost assessment of a new oral care program in the intensive care unit to prevent ventilator-associated pneumonia. Clin Oral Investig. 2018;22(5):1945-51.

27. Brasil. Recomendações AMIB/CFO para atendimento odontológico COVID- 19: Comitê de Odontologia AMIB/CFO de enfrentamento ao COVID-19 Departamento de Odontologia AMIB. Associação de Medicina Intensiva Brasileira. 2020. p. 21.

28. Caruso AA, Del Prete A, Lazzarino AI. Hydrogen peroxide and viral infections: A literature review with research hypothesis definition in relation to the current covid-19 pandemic. Med Hypotheses [Internet]. 2020;144(May):109910. Available from: https://doi.org/10.1016/j.mehy.2020.109910

29. Pattanshetty S, Narayana A, Radhakrishnan R. Povidone-iodine gargle as a prophylactic intervention to interrupt the transmission of SARS-CoV-2. Oral Dis. 2020;(April):1-2.

30. Stucky K, Jutte JE, Warren AM, Jackson JC, Merbitz N. A survey of psychology practice in critical-care settings. Rehabil Psychol. 2016;61(2):201-9.

31. Schneider AM, Moreira MC. Psicólogo intensivista: reflexões sobre a inserção profissional no âmbito hospitalar, formação e prática profissional. Temas em Psicol. 2017;25(3):1225-39. 
32. Coppus ANS, Netto MVRF. A Inserção do Psicanalista em uma Unidade de Tratamento Intensivo. Psicol Ciência e Profissão. 2016;36(1):88-100.

33. Liu Y, Li J, Feng Y. Critical care response to a hospital outbreak of the 2019-nCoV infection in Shenzhen, China. Crit Care. 2020;24(1):4-6.

34. Shen X, Zou X, Zhong X, Yan J, Li L. Psychological stress of ICU nurses in the time of COVID-19. Crit Care. 2020;24(1):2-4.

35. Schmidt B, Crepaldi MA, Bolze DAS, Neiva-Silva L, Demenech LM. Saúde mental e intervenções psicológicas diante da pandemia do novo coronavírus (COVID-19). Estud Psicol. 2020;37:1-13.

36. Castelo Branco AB de A, Arruda KDDSA. Atendimento psicológico de pacientes com COVID-19 em desmame ventilatório: proposta de protocolo. Rev Augustus. 2020;25(51):335-56.

37. Andrade LS de, Andrade LS de, Torres LCD. The importance of the presence of the dental surgeon in the intensive care unit of a municipal hospital in Rio de Janeiro. Acad Rev Científica da Saúde. 2019;04(02):60-4.

38. Tulio K de SC, Stramandinoli-Zanicotti RT, Dirschnabel AJ, Schussel JL, Wasilewskis JHS, Krelling A et al. Alterações no perfil da microbiota bucal durante permanência na UTI : colonização por patógenos respiratórios potenciais Pacientes sob cuidados intensivos constituem um grupo de indivíduos em condições graves , A Unidade de Terapia Intensiva ( UTI ) repre. 2018;7:351-7.

39. Alonso-Herreros JM, Berisa-Prado S, Cañete-Ramírez C, Dávila-Pousa C, Flox-Benítez MDP, Ladrón de Guevara-García M, et al. Hospital Pharmacy Compounding against COVID-19 pandemic. Farm Hosp. 2020;44(7):49-52. 
40. Arney BD, Senter SA, Schwartz AC, Meily T, Pelekhaty S. Effect of Registered Dietitian Nutritionist Order-Writing Privileges on Enteral Nutrition Administration in Selected Intensive Care Units. Nutr Clin Pract. 2019;34(6):899-905.

41. Barazzoni R, Bischoff SC, Krznaric Z, Pirlich M, Singer P. ESPEN expert statements and practical guidance for nutritional management of individuals with SARS-CoV-2 infection. Clin Nutr. 2020;(April):1-8.

42. Sequeira R, Paixão C, Diniz A, Sousa P. Terapia Nutricional nos doentes com COVID19 hospitalizados. Esc Nac Saúde Pública. 2020;

43. Mahanna E, Crimi E, White P, Mann DS, Fahy BG. Nutrition and metabolic support for critically ill patients. Curr Opin Anaesthesiol. 2015;28(2):131-8.

44. Martindale R, Patel JJ, Taylor B, Warren M, McClave SA. Nutrition Therapy in the Patient with COVID-19 Disease Requiring ICU Care. Am Soc Parenter Enter Nutr. $2020 ; 1-8$.

45. Vidal CFDL. Impacto da Higiene Oral com clorexidina com e sem escovação dental na Prevenção da Pneumonia associada à Ventilação Mecânica: Estudo Randomizado Impacto da Higiene Oral com clorexidina com e sem escovação dental na Prevenção da Pneumonia associada à Vent [Internet]. 2014. Available from: https://repositorio.ufpe.br/bitstream/123456789/12053/1/TESE Claudia Fernanda de Lacerda Vidal.pdf\%3E

46. Silva CHF DA, Almeilda RCC de, Benedito FCS, Joaquim DC, Lopes KS, Neto RSM et al. Odontologia Hospitalar: Condições Bucais E Hábitos De Higiene Oral De Pacientes Internados. Braz J Surg Clin Res. 2019;26(1):6-10.

47. Meidani M, Khorvash F, Abbasi S, Cheshmavar M, Tavakoli H. Alloimmunization in 
thalassemia patients: New insight for healthcare. Int J Prev Med. 2018;8:2018-21.

48. Jugend M, Jurkiewicz R. A assistência psicológica através da escuta clínica durante a internação. Rev da SBPH [Internet]. 2012;15(1):3-21. Available from: http://pepsic.bvsalud.org/scielo.php?script=sci_arttext\&pid=S151608582012000100002\&lng=pt\&nrm=iso\&tlng=pt

49. Grincenkov FR dos S. A Psicologia Hospitalar e da Saúde no enfrentamento do coronavírus: necessidade e proposta de atuação. HU Rev [Internet]. 2020;45(1):1-2. Available from: https://doi.org/10.34019/1982-8047.2020.v46.30050\%0A

50. Santana SR de, Girard DCT, Costa LCA da, Girard CMT, Costa DJE da. Os desafios dos serviços psicológicos mediados pelas TIC no contexto da Pandemia do Coronavírus 2019-2020. Rev Bibliotecon e Ciência da Informação. 2020;6(1):59-71. 